\title{
Verbos de apoyo, funciones léxicas y traducción automática
}

\author{
Margarita Alonso Ramos \\ Universidade da Coruña
}

\section{PRESENTACIÓN}

El trabajo de Zarco Tejada que aquí presentamos tiene intención de rellenar un importante vacío en la bibliografía española ${ }^{1}$. Hasta hace poco tiempo eran escasos los trabajos en español (y sobre el español) centrados en las construcciones del tipo dar un paseo, cometer un crimen, echar un vistazo, etc., a las que la autora opta por llamar predicados complejos ${ }^{2}$ (de ahora en adelante, PC). Tampoco son muy abundantes los trabajos en español sobre traducción automática, especialmente centrados en los problemas de transferencia léxica.

1 Zarco Tejada, Ma Ángeles (1998), Predicados complejos y Traducción automática, Cádiz, Servicio de Publicaciones de la Universidad de Cádiz, 285 pp.

2 Hay sin embargo una gran variedad de términos dentro de la bibliografía española: formas descompuestas verbo-nominales (Dubský 1964, 1965a, 1965b), verbos funcionales (Solé 1966), lexías complejas (Cano 1981), perifrasis léxicas (Coseriu 1981), verbos compuestos (Koike 1992, 1996/1997), construcciones verbo-nominales funcionales (Wotjak 1998), verbos soporte (Alonso Ramos 1997), verbos vicarios (Mendívil Giró 1999), etc. En la bibliografía francesa, estas construcciones han sido profundamente estudiadas por el equipo de M. Gross (Giry-Schneider 1987, G. Gross 1989 y 1993, M. Gross 1981, 1994 y 1998, Ibrahim 1996) y por la escuela de I. Mel'čuk de la que hablaremos más adelante. En ambos marcos teóricos son especialmente conocidos por el término de verbe support que algunos adaptamos a verbos de apoyo. En el ámbito inglés, como la autora indica, el término light verb, introducido por Jespersen, suele ser el más utilizado y en ocasiones adaptado al español como verbo liviano (Masullo 1996). 
Cruzar estos dos dominios presenta un gran interés tanto con fines teóricos como con vistas a su implementación computacional. Es sabido que los PC plantean problemas de descripción lexicográfica ya que la información sobre qué verbo selecciona cada nombre debe ser consignada en el artículo lexicográfico del nombre (vid. Hausmann 1979 y 1998, Heid 1992, 1994 y 1996). Todavía desde un punto de vista teórico, los PC suscitan problemas relacionados con la estructura argumental: el predicado se realiza como un nombre y sus argumentos se realizan como sujeto y complementos de un verbo (vid. Grimshaw y Mester 1988). En lo que respecta a la traducción automática, los PC requieren una estrategia de traducción particular: es necesario traducir primero el nombre y después el verbo. En caso contrario, los resultados serían incorrectos. Por ejemplo: la versión francesa del PC dar un paso sería faire un pas (y no *donner un pas); asimismo la versión inglesa sería to take a step (y no *to give <*to make> a step). Una buena descripción teórica y lexicográfica de los PC facilitará por tanto la correcta traducción en un sistema de traducción automática, sin recurrir a la multiplicidad de reglas sensibles al contexto del tipo "traduce dar por faire cuando va con paso".

La autora de este libro se centra en la información léxica que un sistema de traducción automática debe incluir para traducir correctamente los PC en italiano, inglés y español. Los aborda tanto desde un punto de vista paradigmático como sintagmático. Desde el punto de vista paradigmático, le interesa encontrar el formalismo que represente la (cuasi-)sinonimia entre un PC y el verbo asociado, como dar un paseo y pasear, hacer un esfuerzo y esforzarse, etc. Desde el punto de vista sintagmático, busca el medio para controlar la coocurrencia léxica restringida que hace que sea, por ejemplo, dar el verbo seleccionado por $p a-$ seo y no hacer, pero en cambio, el nombre viaje seleccione este último verbo. Para ello, se basa en dos herramientas teóricas muy diferentes, como la misma autora reconoce (p. 5): 1) las estructuras conceptuales, utilizadas en la Semántica conceptual desarrollada por Jackendoff (1983 y 1990), son empleadas para relacionar estructuras superficiales distintas pero con un mismo contenido semántico; 2) las funciones léxicas, herramienta desarrollada por "Mel'čuk" y sus colegas rusos hace 
ya casi cuarenta años en el marco de la Teoría Sentido-Texto 3 , son empleadas para codificar la coocurrencia léxica restringida. A pesar de que estas dos herramientas teóricas tienen ya una sólida tradición y están encuadradas en marcos teóricos bien diferentes, la autora de este libro ha querido fusionarlas e interpretar las funciones léxicas en términos de la Semántica conceptual, lo que la lleva a cometer graves errores. Desafortunadamente, la Teoría Sentido-Texto todavía no es muy conocida en el ámbito español ${ }^{4}$ y no podemos dejar que su difusión se haga alterando la naturaleza de una de sus herramientas básicas, las funciones léxicas. Antes de detallar la confusión reinante en este libro sobre dicha herramienta, debemos presentar la estructura de este trabajo.

El libro consta de una breve introducción y nueve capítulos de extensión e importancia desiguales que agruparé en cinco bloques. El primer bloque, formado por los tres primeros capítulos, nos introduce el objeto de estudio, los PC, el especial interés que estas construcciones presentan en la traducción automática y el corpus con el que la autora ha trabajado. El segundo bloque agrupa los capítulos 4 y 5 , dedicados a exponer las herramientas teóricas que Zarco Tejada ha utilizado para describir los PC. El tercer bloque (capítulo 6) supone la aplicación de estas herramientas a los $\mathrm{PC}$ formados por los verbos prendere y dare y sus correlativos en inglés y español. El bloque cuarto (capítulo 7) constituye la propuesta de este trabajo: vincular las estructuras concep-

3 Sobre presentaciones generales de la Teoría Sentido-Texto, vid. Mel'čuk (1973, 1997b), Steele (1990), Wanner (1997); sobre la sintaxis y la morfología desarrolladas en este marco teórico, vid. Mel'čuk (1988a, 1993, 1994, 1996a y 1997a); sobre semántica y el modelo lexicográfico asociado a la teoría y en particular sobre las funciones léxicas, vid. Apresjan (1992), Mel'čuk (1982, 1988b, 1995, 1996b, 1999), Mel'čuk y Wanner (1996), Mel'čuk et al. (1984, 1988, 1992 y 1999), Mel'čuk et al. (1995), Wanner (1996).

4 No es, con todo, completamente desconocida. Vid. por ejemplo, Alonso Ramos (1993), Luque Durán y Manjón Pozas (1997a y 1997b). Una aplicación de las funciones léxicas al español puede encontrarse en Alonso Ramos $(1989,1991,1993$, 1994-1995 y 1997). Para un esbozo de un diccionario bilingüe, véase Lépinette (1989). Sobre la descripción lexicográfica de los nombres de emoción en español, vid. Sanromán (1998 y 1998-1999). 
tuales con las funciones léxicas por medio de la estructura argumental, lo que conlleva, como veremos, una desnaturalización de las funciones léxicas. El último bloque, formado por los capítulos 8 y 9 , queda un tanto desligado del resto. El capítulo 8, que debería haber aparecido antes como presentación del estado de la cuestión, expone el tratamiento que han tenido los $\mathrm{PC}$ en otros léxicos computacionales. Y, por último, el capítulo 9, a modo de anexo o apéndice, incluye las entradas lexicográficas del español, inglés e italiano, correspondientes a los PC y a los verbos asociados. Se echa en falta un capítulo en donde se recojan las conclusiones del trabajo.

En cuanto al aspecto formal, no se puede decir que se haya extremado el rigor. Son muchos los términos que la autora deja en inglés, a pesar de que han sido ya traducidos al español en otros trabajos. Por ejemplo, los nombres de los papeles semánticos aparecen siempre en inglés ("Agent" "Theme" etc.). Asimismo, el nombre de la teoría lingüística Teoría Sentido-Texto aparece siempre mencionada por su denominación en inglés. También existen algunos errores tipográficos u ortográficos como "prédicado" (p. 40), "fusinada" (p. 113) o "Mel'cuk", que en una revisión más reposada del texto se habrían detectado. Lo que consideramos más reprochable es el gran número de referencias de segunda mano. En pocas ocasiones, la autora de este trabajo ha ido a las fuentes primeras y cita a menudo por fuentes indirectas. Por ejemplo, cita el trabajo de Smadja (1993), publicado en una revista de amplia difusión, por medio de Heylen (1993), que, sin embargo, es el informe de un proyecto de investigación holandés, mucho menos accesible para el lector español. Igualmente reproduce una cita de Steele y Meyer (1990), libro publicado por la Universidad de Ottawa, a través de Bloksma et al. (1993), que es otro informe del mencionado proyecto. También Heid y Raab (1989), trabajo publicado en las Actas de la Association of Computational Linguistics, es citado a través de otro informe del mismo proyecto, Balkan (1993).

Pasemos ya a una exposición crítica del contenido del trabajo de Zarco Tejada. 


\section{EXPOSICIÓN CRÍTICA}

El capítulo 1 “Qué son los predicados complejos?” enmarca los PC como un tipo especial de colocación, siguiendo especialmente a Allerton (1984) y a Aisenstadt (1979) ${ }^{5}$. A pesar de las diversas citas que la autora reproduce, en ningún momento llega a formular una definición del concepto de colocación. En las páginas 10 y 11, se señala que el término colocación se refiere a la relación léxica entre dos elementos, la base y el colocante. El primero sería el elemento seleccionador y el segundo, el elemento seleccionado. Así, en el caso de los PC, el nombre sería la base y verbo el colocante (también llamado por otros autores colocativo). Ahora bien, la autora usa indistintamente los términos selección y rección para explicar la especial relación léxica entre los dos elementos de una colocación. Si bien es cierto que el término rección tiene distintos usos en lingüística, el más usual refiere a una relación sintáctica y no léxica. No consideramos, por tanto, adecuado decir que en una colocación dada el sustantivo rige un verbo determinado y no otro. El verbo rige sintácticamente al sustantivo como en cualquier otro sintagma libre, pero es el nombre el que selecciona léxicamente al verbo.

El término restricción léxica aparece también en la distinción entre las colocaciones y las expresiones idiomáticas. Para la autora, ambos tipos de combinaciones de palabras coinciden en la restricción o selección léxica que se produce entre los elementos que la forman (p. 15). Podría pensarse que el grado máximo de coocurrencia léxica restringida lo encontramos en las expresiones idiomáticas. En efecto, si la combinación entre echar y firma para significar 'firmar' es hasta cierto punto arbitraria, la arbitrariedad es mucho mayor en la combinación entre echar y guante para significar 'apresar'. Sin embargo, discrepamos de este punto de vista que puede llevar a cierta confusión. El ha-

5 Curiosamente, no menciona en ningún momento una definición de colocación emanada de la Teoría Sentido-Texto, a pesar de que opta por tratar las colocaciones por medio de la herramienta de esta teoría. Vid. por ejemplo, Alonso Ramos (19941995), Mel'čuk (1995 y1998), Wanner (1996), etc. 
blante no combina los lexemas echar y guante para producir un sentido dado sino que elige una expresión compacta, ya construida en la lengua. Las expresiones idiomáticas como echar el guante o el célebre estirar la pata constituyen unidades léxicas, a pesar de que están formadas por varias palabras. La razón principal por la cual las expresiones multilexémicas (= locuciones o expresiones idiomáticas) y las palabras (= lexemas) deben tener artículos lexicográficos es que ambas son no composicionales. Sin embargo, las colocaciones están constituidas por dos unidades léxicas, una de las cuales selecciona a la otra sin que haya una razón semántica obvia de por qué una base selecciona un colocativo dado y no otro. La composicionalidad de las colocaciones, como la propia autora señala, es el factor principal para su distinción de las expresiones idiomáticas.

Hacia el final de este capítulo, los PC quedan descritos por tres condiciones necesarias: 1) se trata de sintagmas ("estructuras") en donde se da algún grado de restricción léxica; 2) su significado es composicional; y 3) el sintagma está constituido por "V + Complemento". En el "amplio cajón de los complementos" (p. 29), la autora incluye tanto la estructura $\mathrm{V}+\mathrm{SN}$ como $\mathrm{V}+\mathrm{SP}$. Tras un somero repaso a la teoría de la valencia, la autora opta por tratar los complementos de los PC como complementos integrales (término utilizado por Somers 1984), que se caracterizan por ofrecer resistencia a posibles omisiones debido a que son parte integral del predicado (p. 42). A diferencia de los complementos obligatorios como los del verbo inglés to watch que pueden pertenecer a una clase semántica (I watched the footbal match $<$ the view, the film, $T V>$ ), los complementos integrales están léxicamente determinados.

Si el primer capítulo se ocupa del eje sintagmático de los PC, el capítulo 2 "La correspondencia estructural en traducción automática" se centra en el eje paradigmático. Se trata de subrayar que un mismo contenido semántico puede expresarse por medio de un PC (cometer un error) o por una unidad léxica simple (equivocarse). Esta doble posibilidad de realización es especialmente interesante en el ámbito de la traducción automática ya que una lengua puede tener ambas posibilidades, mientras que otra puede contar sólo con una. La autora sintetiza 
las tres posibilidades lógicas: 1) PC $\rightarrow \mathrm{PC}$ (dar un paseo $\rightarrow$ to take a walk); 2) PC $\rightarrow$ Verbo (poner la zancadilla $\rightarrow$ to trip up); y 3 ) Verbo $\rightarrow$ PC (suicidarse $\rightarrow$ to commit suicide). Este será el objeto de interés de la autora: la correspondencia o falta de correspondencia entre PC y verbos de tres lenguas, italiano, inglés y español. La lengua de origen de la que parte es el italiano y para ello, ha tomado el Corpus del Instituto di Linguistica Computazionale de Pisa, que presenta en el capítulo 3. De ese corpus ha seleccionado los PC constituidos por los verbos mettere, prendere y dare que serán estudiados en los capítulos siguientes con el objetivo de encontrar un modo de representación que permita traducirlos al español y al inglés.

El segundo bloque engloba los capítulos 4 y 5 . Ambos exponen las herramientas teóricas con que la autora se dispone a describir los PC en un léxico con vistas a su utilización en un sistema de traducción automática. El capítulo 4 "Representación conceptual del nivel paradigmático" expone la Semántica conceptual, desarrollada por Jackendoff (1983) y (1990). Las estructuras conceptuales à la Jackendoff sirven a la autora para intentar mostrar que un mismo contenido semántico puede realizarse por medio de un verbo simple o por medio de una colocación. Siguiendo el ejemplo de Jackendoff (1990), to butter y to put some butter on, en este capítulo se hace un somero análisis de verbos en italiano, inglés y español que incorporan bien el argumento Tema, bien el argumento Meta. Por ejemplo: taponar, anillar, archivar, encarcelar, etc. Todos ellos son parafraseados por el verbo poner (o su correspondiente en italiano e inglés para los equivalentes de estos verbos en dichas lenguas). Así, taponar es parafraseado por 'poner el tapón en algo' o archivar como 'poner algo en un archivo'. Sin embargo, estas paráfrasis son más de índole metalingüística que propiamente lingüística. Si queremos que un sistema nos traduzca un verbo como to butter, que no tiene equivalente monolexémico en español, el léxico debe proporcionar la colocación untar algo de mantequilla y no poner mantequilla sobre algo. Lo mismo ocurre con otros ejemplos: el equivalente colocativo de taponar es cerrar algo con un tapón, el de anillar es sujetar algo con anillas, el de archivar es guardar algo en un archivo, el de encarcelar es meter a alguien en la cárcel, etc. (vid. Sanromán et 
al. 1999 para más ejemplos sobre verbos denominales y colocaciones relacionadas). Disentimos de la conclusión de la autora cuando señala que "la aproximación conceptual, como medio para relacionar oraciones con el mismo significado, pero diferente estructura sintáctica, es muy conveniente" (p. 95). No ponemos en duda que la estructura conceptual puede ser común a diferentes estructuras sintácticas, pero sí que la paráfrasis metalingüística sea válida como equivalentes de traducción. Nótese la extrañeza de $A$ Juan lo *pusieron <metieron, encerraron> en la cárcel.

La segunda herramienta teórica queda expuesta en el capítulo 5 "Las funciones léxicas de Mel'čuk". Tras una brevísima introducción de página y media a la Teoría Sentido-Texto, marco teórico en el que operan las funciones léxicas (de ahora en adelante, FFLL), se nos reproducen cuatro definiciones, más o menos equivalentes, del concepto de FL, sin que en ningún momento la autora sintetice y formule en sus propios términos una definición. Las FFLL constituyen una herramienta lexicográfica ideada para describir las relaciones léxicas paradigmáticas y sintagmáticas del lema de un artículo lexicográfico. Por medio de un símbolo especial, una FL asocia con una unidad léxica dada $\mathbf{L}$ otra unidad léxica (o conjunto de unidades léxicas) $\mathbf{L}$ ' que sostiene con respecto a $\mathbf{L}$ una relación semántica paradigmática o sintagmática. Una FL se presenta como una función en el sentido matemático, en donde $\mathbf{L}$ es el argumento de la función y $\mathbf{L}$ ', su valor: FL $(\mathbf{L})=\mathbf{L}$ '.

La autora se ha centrado especialmente en las FFLL que describen relaciones sintagmáticas y más en particular, las que describen colocativos verbales. Siguiendo especialmente a Mel'čuk y Žolkovskij $(1970)^{7}$, la autora reproduce las definiciones de algunas FFLL verba-

6 No puede dejar de llamarnos la atención la extrañeza de este título. Mel'čuk junto con otros colegas rusos fue el creador de la herramienta lexicográfica de las funciones léxicas, ahora bien, dentro de una teoría lingüística, la Teoría Sentido-Texto y más en particular, dentro de un modelo lexicográfico, el Diccionario explicativo y combinatorio. Vid más abajo las referencias bibliográficas.

7 Han pasado casi treinta años de la publicación de ese trabajo y hay muchas actualiza- 
les. Le interesa particularmente la FL Oper, que es la encargada de asociar un nombre predicativo con un verbo semánticamente vacío. Los ejemplos en inglés proceden del artículo antes mencionado:
$\operatorname{Oper}_{1}($ blow $)=$ to strike
$\mathrm{Oper}_{2}($ visit $)=$ to receive, to have
$\operatorname{Oper}_{1}($ step $)=$ to take
$\operatorname{Oper}_{2}($ humiliation $)=$ to undergo
Oper ${ }_{1}($ order $)=$ to give
$\operatorname{Oper}_{3}($ order $)=$ to have

Aunque la autora no llega a aclarar el papel de los índices numéricos, lo que desencadenará un sinfín de reinterpretaciones de las FFLL en los siguientes capítulos, es necesario decir que el índice numérico de la FL refiere al actante sintáctico profundo del nombre. Así, el nombre blow tiene cuatro actantes sintácticos profundos: blow by $X$ to $Y$ on the bodypart $Z$ with $W$. Cada uno de ellos designa un participante en la situación descrita por el nombre predicativo. La numeración de los actantes sigue el orden de realización lingüística. Así, el actante sintáctico profundo 1 alude a X, el actante sintáctico profundo 2 a Y, etc. En el caso de la FL $\mathrm{Oper}_{1}$, el primer actante sintáctico profundo del nombre será el sujeto gramatical del valor y en el caso de $\mathrm{Oper}_{2}$, será el segundo actante sintáctico profundo del nombre el que funcione como sujeto gramatical del verbo. Así con order, que tiene la siguiente forma proposicional order by $X$ to $Z$ to do $Y$, en to give an order, es $X$ quien funciona como sujeto del verbo to give, mientras que en to have an order, es $Z$ quien desempeña ese papel.

Existe también otra FL $\mathrm{V}_{0}$ que describe el verbo cuasi-sinónimo de la palabra en cuestión. Por ejemplo:

$$
\mathrm{V}_{0}(\text { assistance })=\text { to assist }
$$

En este caso, la relación establecida es paradigmática y por lo tanto, el valor de la FL no se combina con el argumento o palabra llave. Algunas FFLL sintagmáticas tienen expresiones fusionadas, es decir, valores que no se combinan con la palabra llave de la FL. Por ejemplo, la

ciones de la teoría y adaptaciones al español, que la autora parece desconocer. Vid. las referencias bibliográficas en las notas 2,3 y 4 . 
FL sintagmática Magn cuyo sentido aproximado es 'muy' o 'intenso' puede tener valores que cubran el significado de la propia FL y el de la palabra llave. Es el caso de memorión que significa 'una memoria muy grande' y por lo tanto, corresponde a la FL Magn(memoria). Sin embargo, no puede combinarse con memoria ya que el sentido 'memorión' incluye el sentido 'memoria'. Cuando hay valores fusionados, se indica a través del signo //. Así, en la entrada de memoria, tendremos:

$\operatorname{Magn}($ memoria $)=$ portentosa, prodigiosa, de elefante $/ /$ memorión .

Ahora bien, la autora interpreta $\mathrm{V}_{0}$ como la expresión fusionada de la FL Oper y consigna el valor de la FL $\mathrm{V}_{0}$ tras las dos barras diagonales. En el caso del nombre assistance, la notación sería la siguiente (p. 114):

$\operatorname{Oper}_{1}($ assistance $)=$ to provide $/ /$ to assist

Se trata claramente de una falta de comprensión del funcionamiento de la FL Oper. Si esta FL describe un verbo semánticamente vacío, no puede tener como valor una expresión fusionada ya que ésta debe incluir el significado de la propia FL, y Oper, como tal, no tiene ningún significado sino que representa simplemente un verbo de apoyo sintáctico.

La presentación de las FFLL termina con una crítica de esta herramienta achacándole ciertas imprecisiones como el concepto de actante sintáctico profundo, que la autora no ha entendido. La razón de estas imprecisiones es debida, según la autora, a que esta teoría lingüística nació del ruso, lengua nativa de los creadores, y que los ejemplos no se han adaptado bien a otras lenguas (p. 116). Resulta sorprendente esta crítica cuando la Teoría Sentido-Texto y en concreto, las FFLL han sido aplicadas ya a cuatro volúmenes del Dictionnaire explicatif et combinatoire du français contemporain, así como a diferentes publicaciones en inglés y en español, que la autora parece desconocer (vid. Mel'čuk et al. 1984, 1988, 1992 y 1999).

El tercer bloque está formado por el capítulo 6 "Representación interlingual de los niveles sintagmático y paradigmático", que constituye la aplicación de las herramientas teóricas expuestas en los dos capítu- 
los precedentes. Aquí la autora parte de los verbos italianos prendere y dare y sus correlativos en inglés y español, formantes de PC y estudia 18 verbos equivalentes semánticamente a esos $\mathrm{PC}$. Su ejemplo recurrente es el par tomar alojamiento y alojarse, a los que atribuye la misma estructura conceptual, en términos de Jackendoff. En lo que respecta al nivel sintagmático, empieza por describir la relación entre tomar y alojamiento por medio de la FL $\mathrm{Oper}_{2}$ y el par dar y alojamiento por medio de Oper:

$\operatorname{Oper}_{2}($ alojamiento $)=$ tomar

$\operatorname{Oper}_{1}($ alojamiento $)=$ dar

Sin embargo, se le presenta el problema de cómo describir recibir alojamiento. Si representa esta colocación por medio de $\mathrm{Oper}_{2}$, se da el caso de que dos estructuras conceptuales diferentes serían descritas por una misma FL. Asimismo, si tomar alojamiento es descrito por la FL $\mathrm{Oper}_{1}$, la misma FL representaría la estructura conceptual de dar alojamiento, que es diferente de la de tomar alojamiento.

En este capítulo, la autora opta por las formulaciones siguientes ( $\mathrm{p}$. 134):

Oper ${ }_{1}($ alojamiento $)=$ dar $/ /$ alojar

$\mathrm{Oper}_{2}($ alojamiento $)=$ recibir

Oper $($ alojamiento $)=$ tomar $/ /$ alojarse

Como vemos, el último par queda sin índice actancial, en espera de identificar más tarde cuál será la relación temática del sujeto de tomar. Los problemas de asignación de FFLL a estos predicados complejos surgen debido a que la autora interpreta los índices actanciales por medio de las relaciones temáticas de Jackenfoff. Así, interpreta el índice 2 como un "afectado" o "beneficiario" (p. 130) y el índice 1 como un "actor" (p. 132). Nada más lejos de la interpretación que tienen estos índices en la Teoría Sentido-Texto, en donde los papeles temáticos no desempeñan ninguna función. A lo largo de todo el capítulo, el más largo del libro, la autora examina pares como tomar un riesgo-arriesgarse, tomar distancia-distanciarse, tomar asiento-sentarse, echar la culpa-culpar, dar asistencia-asistir, etc. y va formulando las estructu- 
ras conceptuales correspondientes y asignando FFLL con los criterios confundidos.

El cuarto bloque constituye la propuesta de este trabajo. En el capítulo 7, titulado "Nueva definición de Función Léxica", se nos ofrece una reinterpretación de los índices actanciales de las FFLL y una nueva definición, no del concepto de FL, como el título sugiere, sino una nueva definición de la FL Oper. Retomando los problemas sin resolver con respecto a la asignación de FL para describir tomar alojamiento, empieza con un estudio crítico de las FFLL Oper y Oper $_{2}$. En la página 182, falsea la descripción de la teoría al afirmar categóricamente que "según Mel'čuk los subíndices 1 y 2 (...) equivalen a una especie de Agent y Experiencer". Esta interpretación incorrecta la lleva a un callejón sin salida. Si el índice 1 describe un "Agent", Oper ${ }_{1}$ describiría correctamente la colocación dar alojamiento, pero no tomar una decisión porque, según la autora, el sujeto gramatical tendría el papel semántico ("role temático", en sus términos) de "Source" y no de "Agent". Si el índice 2 es una especie de "Experiencer", no habría manera de distinguir entre tomar alojamiento y recibir alojamiento. Para solventar estos problemas, la autora recurre a los "roles de acción" del tipo "Actor/Afectado". Decide asignar la FL Oper ${ }_{2}$ a los PC cuyo sujeto gramatical tenga el rol "Afectado", como tomar color; la FL Oper será asignada cuando el sujeto gramatical tenga el rol "Actor", como tomar una decisión. Los casos problemáticos como tomar alojamiento $<$ un riesgo, distancia $>$ quedan descritos por medio de un nuevo índice Oper $_{1-2}$. En estos casos, el sujeto gramatical tiene el papel semántico "Theme" y la doble posibilidad de desempeñar ambos roles de acción "Actor/<Afectado".

Por medio del concepto de estructura argumental, tal y como lo formulan Rappaport y Levin (1988), la autora pone en relación las estructuras conceptuales con las FFLL. Así, a partir de la estructura conceptual de un PC, deriva una estructura argumental y formula una regla de asignación de FL. Por ejemplo, la estructura conceptual de dar alojamiento queda descrita por las siguientes sub-estructuras:

a. [ $\mathrm{x}$ causes an action on $\mathrm{y}]$ 

b. [y comes to be at location]
c. [ $\mathrm{z}$ is a location]

Por medio de ciertas reglas de relación ("linking rules"), la estructura argumental derivada es: DAR: $\mathbf{x}<\mathbf{y}, \mathbf{P z}>$. En dicha estructura, no queda claro qué variable argumental está representando a alojamiento. Según la estructura conceptual, la variable y alude al participante que cambia de situación (al 'alojado'). Sin embargo, en la estructura argumental, la variable y refiere al argumento directo, es decir, al objeto directo. Falta, por tanto, una variable argumental ya que si bien semánticamente 'dar alojamiento' constituye un todo semántico, desde el punto de vista sintáctico, alojamiento está desempeñando una función sintáctica: el verbo dar rige sintácticamente a su sujeto gramatical (x), a su complemento directo (alojamiento), a su complemento indirecto (y) y a un complemento circunstancial (un SP como en su casa).

Menos clara es todavía la estructura conceptual propuesta para tomar una decisión. Véase:

a. [y comes to be FROM w]

b. [w is a locator]

La consiguiente estructura argumental es: TOMAR: $\mathbf{w}<\mathbf{y}>$.

Sea como sea, la estructura argumental sirve a la autora para formular un regla de asignación de FL. Así, para poder describir un PC por medio de $\mathrm{Oper}_{1}$, es necesario que el índice 1 represente a la variable $\mathbf{x}$ o a la variable $\mathbf{w}$ y que se corresponda conceptualmente con un "Actor". Formulaciones similares a éstas son propuestas para la asignación de $\mathrm{Oper}_{2}$ y de la "nueva" Oper ${ }_{1-2}$. De una manera un tanto simplista, la autora concluye afirmando que "hemos dado una fórmula a lo que antes sólo se había definido con palabras" (p. 204).

En la sección siguiente, ofreceré una clarificación del concepto de FL para contrastar los análisis de la autora con la práctica habitual del uso de esa herramienta lexicográfica, tal y como se emplea en la Teoría Sentido-Texto. 


\section{QUÉ SON REALMENTE LAS FFLL}

Dada la escasa difusión de la Teoría Sentido-Texto en el ámbito hispano, no podemos dejar pasar la oportunidad de aclarar ciertos malentendidos sobre las FFLL que el trabajo de Zarco Tejada transluce. Como ya hemos señalado, las FFLL constituyen una herramienta lexicográfica ideada para describir las relaciones léxicas paradigmáticas y sintagmáticas del lema de un artículo lexicográfico. Las FFLL no son, por tanto, una herramienta desligada de la descripción lexicográfica completa de una unidad léxica dada. El artículo lexicográfico de un diccionario del tipo Diccionario explicativo y combinatorio $(=\mathrm{DEC})$ incluye una zona semántica en donde se describe con rigor la definición del lema, tomando como punto de partida la forma proposicional. Así, el definiens de la definición del nombre pregunta sería: la pregunta de $X$ sobre $Y$ a $Z$. Las variables actanciales designan los participantes en la situación descrita por el nombre pregunta y constituyen sus actantes semánticos. En ningún momento, los actantes semánticos son etiquetados por medio de etiquetas del tipo "Agent" o "Experiencer" o "Theme" ya que la propia definición describe cuál es la relación semántica que cada uno de los actantes desempeña con respecto al predicado. Simplemente los actantes son numerados siguiendo el orden de realización en la superficie. Así, el participante X, que desempeñaría el sujeto gramatical en la versión verbal de este predicado, será considerado el actante sintáctico profundo I; el actante Y, que desempeñaría el complemento directo, será considerado el actante II y así sucesivamente. La correspondencia entre los actantes semánticos y los actantes sintácticos profundos se representa en una zona especial del artículo lexicográfico, bajo la forma de una tabla llamada "Régimen". Véase:

Tabla I. Régimen de pregunta

\begin{tabular}{|c|c|c|}
\hline$X=I$ & $\mathrm{Y}=\mathrm{II}$ & $\mathrm{Z}=\mathrm{III}$ \\
\hline $\begin{array}{l}\text { 1. de } \mathrm{N} \\
\text { 2. } A_{p o s}\end{array}$ & $\begin{array}{l}\text { 1. sobre N } \\
\text { 2. de si Prop } \\
\text { 3. sobre si Prop } \\
\text { 4. de Pron } \\
\text { interog }\end{array}$ & 1. a N \\
\hline
\end{tabular}


En cada una de las columnas, se consignan las realizaciones superficiales de cada uno de los actantes. Por ejemplo: la pregunta de Juan (I) sobre María (II) a Pepe (III); su (I) pregunta sobre si vendría pronto (II); la pregunta de Juan (I) a Pepe (III) de qué hacía aqui (II).

Tanto la zona semántica (la definición) como la zona sintáctica (el régimen) están en estrecha relación con la zona de FFLL, que consigna las relaciones paradigmáticas y sintagmáticas que el lema sostiene. Así por ejemplo, en la entrada de pregunta, se consignarán, por una parte, sus (cuasi-)sinónimos, antónimos, conversivos, derivados semánticos y sintácticos (demanda, cuestión, respuesta, contestación, interrogatorio, interrogador, preguntante, preguntón, etc.) y por otra parte, todas las unidades léxicas que coocurren con pregunta y que forman colocaciones. Por ejemplo: hacer, formular, contestar, responder, satisfacer, dejar en el aire, achicharrar <acribillar, acosar > a, arriesgar <aventurar >, sortear <soslayar >, directa, capciosa, retórica, impertinente, entrometida, inoportuna, chaparrón <diluvio, sinfin $>$ de, etc. Por medio de un símbolo especial que representa el sentido y el comportamiento sintáctico del valor de la FL, del lado paradigmático, tendremos:

$\operatorname{QSyn}($ pregunta $)=$ demanda, cuestión

$\mathrm{Conv}_{321}($ pregunta $)=$ respuesta, contestación

Mult $($ pregunta $)=$ interrogatorio

$\mathrm{V}_{\mathrm{o}}($ pregunta $)=$ preguntar

$\mathrm{S}_{1}($ pregunta $)=$ preguntante

$\mathrm{Able}_{1}($ pregunta $)=$ preguntón

Como muestra de algunas FFLL que describen colocaciones, tenemos:

Oper $($ pregunta $)=$ hacer, formular $[\mathrm{ART} \sim]$

Func $_{2}($ pregunta $)=$ versa $[$ sobre $\mathrm{N}=\mathrm{Y}]$

Func $_{3}($ pregunta $)=$ se dirige $[\mathrm{a} \mathrm{N}=\mathrm{Z}]$

$\mathrm{Real}_{3}($ pregunta $)=$ contestar, satisfacer, responder $[\mathrm{ART} \sim]$

AntiBon + Labor $_{13}=$ achicharrar, acribillar, acosar $[$ a $\mathrm{N} \mathrm{a} \sim \mathrm{s}]$

sin meditar, Oper $_{1}=$ aventurar, arriesgar [ART $\sim$ ]

intentar nonReal ${ }_{3}=$ sortear, soslayar [ART ]

AntiBon $_{2}$ (pregunta $)=$ impertinente, entrometida, inoportuna

Magn + Mult(pregunta $)=$ chaparrón $<$ diluvio, $\operatorname{sinfin}>d e[\sim \mathrm{s}]$ 
Sin comentar cada una de las FFLL aquí ejemplificadas porque nos saldríamos del tema de este trabajo, sí que me gustaría exponer con algo de detalle la función de los índices numéricos. Como acabamos de ver, los índices aparecen tanto en las FFLL paradigmáticas como en las sintagmáticas y en todos los casos, hacen referencia a los actantes sintácticos profundos de la palabra llave, en nuestro caso, pregunta. Por ejemplo, el índice 1 en $\mathrm{S}_{1}$ nos describe el nombre típico del primer actante de pregunta; en $\mathrm{Able}_{1}$, nos proporciona el atríbuto típico del que tiene tendencia a ser primer actante de pregunta. En la FL Conv ${ }_{321}$, los índices sirven para dar cuenta de la permutación que presentan los actantes con respecto a el nombre pregunta: si X es el primer actante de pregunta, será el tercer actante de respuesta y asimismo, si $\mathrm{Z}$ es el tercer actante de pregunta, será el primero de respuesta (la respuesta de $Z$ sobre $Y a X)$.

En las FFLL sintagmáticas, vemos que el índice de Oper ${ }_{1}$ no hace referencia ni al "Actor" ni al "Experiencer", conceptos completamente extraños a esta teoría. Simplemente, esta FL nos proporciona el verbo con el que se combina pregunta tomando como sujeto gramatical el primer actante, es decir, el 'inquisidor' y pregunta como complemento directo de este verbo. El valor de la FL Real ${ }_{3}$ también toma pregunta como complemento directo, pero, en este caso, el sujeto gramatical del verbo será el actante III, el 'destinatario'. En el caso de Func ${ }_{2}$ es pregunta el que funciona como sujeto gramatical del valor de la FL y el primer complemento será el actante II, es decir, 'lo preguntado'. La misma estructura sintáctica presenta Func ${ }_{3}$, con la diferencia de que el primer complemento del verbo será el actante III, el 'destinatario' de la pregunta.

Como vemos, poco tienen que ver los índices actanciales de las FFLL con los papeles temáticos o de acción, derivados de la Semántica conceptual. Lo que reprochamos específicamente a este trabajo es la crítica a la herramienta de las FFLL con los presupuestos teóricos de otra teoría. Evidentemente, no se puede pretender que las FFLL respondan a los mismos criterios semánticos utilizados en la Semántica conceptual y acusar a esta herramienta de "falta de claridad" (p. 116). Y no es que carezcan de imprecisión, pero ésta debe ser medida de acuerdo con los criterios de la teoría de la que las FFLL forman parte. 
Los problemas de asignación de FFLL a ciertos PC, que se le plantean a la autora, se deben a su intención de traspasar conceptos como "Agent", "Theme", "Actor" o "Afectado" a una herramienta en donde no tienen cabida. Así, independientemente de que el sujeto del PC tomar color sea o no "Afectado", la FL que lo describe no es Oper, ${ }_{2}$, sino IncepOper. ${ }_{1}$ La mayoría de los PC con el verbo tomar añaden un valor incoativo, representado por medio de la FL Incep. Para poder asignar una FL, es necesario partir de una definición del nombre, aunque sea aproximada, y determinar cuál es su forma proposicional y, por tanto, cuántos actantes tiene. El nombre color tiene dos actantes semánticos, el objeto que manifiesta la propiedad del color y el valor del color (rojo, azul, etc.). Por lo tanto, la forma proposicional será el color $Y$ de $X$. El referente del sujeto de la colocación tomar color alude a X, es decir, al objeto que manifiesta la propiedad del color. La colocación dar color no sería tampoco descrita por Oper (vid. p. 230) sino por una FL

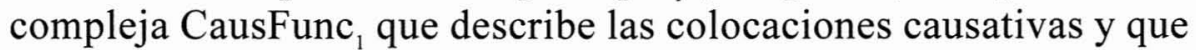
puede ser parafraseada por 'alguien causa que $\mathrm{X}$ tenga color'.

Las mismas críticas merecen otras muchas asignaciones erróneas de FFLL. Tampoco es necesario verificar si el sujeto de tomar una decisión es o no es un "Actor". Ni si el índice actancial 1 se unifica con la variable argumental $\mathbf{w}$ propuesta por la autora. La descripción por medio de la FL IncepOper, se basa en la forma proposicional del nombre decisión: decisión de $X$ de (hacer) $Y$. Se trata de un nombre de dos actantes, 'el que decide' y 'lo decidido' y se toma el primero como actante I y el segundo como actante II.

No es tampoco útil la "nueva" FL Oper $_{1-2}$ para los PC que a la autora no le encajan en ninguno de los papeles semánticos, como tomar alojamiento. A la autora se le escapa la distinción semántica entre dos unidades léxicas alojamiento: una que al igual que su verbo sinónimo alojarse tiene dos actantes: alojamiento 1 de $X$ en $Y$; y otra que al igual que su verbo sinónimo alojar tiene tres actantes: alojamiento 2 de $X a$ $Y$ en $Z$. De esta manera, la colocación tomar alojamiento sería descrita por la FL IncepOper ${ }_{1}$ (alojamiento 1), mientras que dar alojamiento sería tratada como Oper $_{1}($ alojamiento 2$)$. Con respecto a recibir aloja- 
miento, que no suena del todo natural, al menos en mi idiolecto, la FL apropiada sería $\operatorname{Oper}_{2}$ (alojamiento 2).

\section{CONCLUSIÓN}

A pesar de las abundantes inexactitudes con respecto a las FFLL, debidas probablemente a un desconocimiento de la bibliografía al respecto, es de destacar el interés que presenta la idea original de este trabajo. La utilización de un formalismo que dé cuenta de la relación de paráfrasis entre los $\mathrm{PC}$ y los verbos simples, junto con otro formalismo especialmente diseñado para atender a la coocurrencia léxica restringida entre los miembros de cada PC es de gran utilidad en todo léxico que pretenda ser utilizado en un sistema de procesamiento automático de lengua natural. No sólo en sistemas de traducción automática sino también en los de análisis y especialmente, en los de generación automática se ha comprobado la necesidad de desarrollar el componente léxico y de enriquecerlo con la mayor información posible. Aplaudimos por tanto la idea original de la autora, pero no podemos estar de acuerdo con el enfoque propuesto, como creo haber mostrado más arriba. Si es cierto que las FFLL han de ser mejor perfiladas en diferentes aspectos, especialmente con vistas a su implementación computacional, esa tarea ha de llevarse a cabo partiendo de los presupuestos teóricos de los que forman parte. Sin quitar mérito alguno a la Semántica conceptual, tal y como la ha desarrollado Jackendoff, no puede ser esta teoría la que sirva para perfilar mejor el formalismo de las FFLL, ya que parten de premisas teóricas dispares.

Sólo nos queda por señalar que esperamos que este trabajo despierte, con todo, el interés del lector sobre el interesante mundo de las colocaciones, las FFLL y la traducción automática. 


\section{REFERENCIAS BIBLIOGRÁFICAS}

AISENSTADT, E. (1979): “Collocability Restrictions in Dictionaries", en R. R. K. Hartman, (ed.), Dictionaries and Their Users. Papers from the 1978 B.A.A.L. Seminar on Lexicography. Exeter Linguistics Studies, Volume 4. University of Exeter, pp. 71-44.

Allerton, D. J. (1984): "Three (or Four) Levels of Word Cooccurrence Restriction", Lingua, 63: 1, pp. 17-40.

Alonso Ramos, M. (1989): "Aproximación a un nuevo modelo lexicográfico: el Dictionnaire explicatif et combinatoire du français contemporain. Recherches lexico-sémantiques de Igor Mel'čuk", Verba, 16, pp. 421-450.

(1991): "Verbes supports et Fonctions lexicales", Linguisticae Investigationes, XV:1, pp. 203-223.

(1993): Las funciones léxicas en el modelo lexicográfico de I. Mel'cuk, Tesis de doctorado, Madrid, U.N.E.D.

—_ (1994-1995): "Hacia una definición del concepto de colocación: de J. R. Firth a I. Mel'čuk", Revista de Lexicografia, 1, pp. 9-28.

(1997): “Cooccurrencia léxica y descripción lexicográfica del verbo DAR: hacia un tratamiento de los verbos soportes", Zeitschrift für Romanische Philologie, 113: 3, pp. 380-417.

APRESJAN, J. (1992): Lexical Semantics. User's Guide to Contemporary Russian Vocabulary, Ann Arbor, Karoma.

BALKAN, L. (1993): "Review Existing Systems", Collocations ET-10/75, vol. 1, pp. 259-285.

Bloksma, L., D. Heylen y K. G. Maxwell (1993): “Analysis of Lexical Functions", Collocations ET-10/75, vol. 1, pp. 49-69.

DANLOS, L. (1994): "Coder des informations monolingues sur les noms pour éviter des règles bilingues sensibles au contexte", Langages, 116 , pp. 95-110.

DUBSKÝ, J. (1963): "Las formas descompuestas en el español antiguo", Revista de Filología Española, 66, pp. 31-48.

- (1964): "El campo sintagmático de las formas descompuestas en español", Revista de la Universidad de la Habana, 168-169, pp. 109125.

(1965a): “Composition, dérivation et décomposition", Lingua, 16, pp. 190-198.

(1965b): "Intercambio de componentes en las formas descompuestas españolas", Bulletin Hispanique, 77, pp. 343-352. 
(1984): "El valor explícito de las construcciones verbales y verbonominales del español”, Español Actual, 41, pp. 13-20.

(1990): "El campo léxico de los verbos de introducción, de relación, de apoyo y verbos formemáticos", en G. Wotjak y A. Veiga (eds.), La descripción del verbo español, Santiago de Compostela, Universidad de Santiago de Compostela (Verba, Anexo 32), pp. 107-111.

_ (1998): "Debilitamiento de valor comunicativo del verbo español", en G. Wotjak (ed.), pp. 239-244 [reproducción del artículo publicado en Leipziger Linguistische Arbeitsberichte, 45]

GIRY-SCHNEIDER, J. (1987): Les prédicats nominaux en français: les phrases simples à verbe support, Genève/Paris, Droz.

Grimshaw, J. y A. Mester (1988): "Light Verbs and $\theta$-Marking", Linguistic Inquiry, 19, 2, pp. 205-232.

Gross, G. (1989): Les constructions converses du français, Genève/Paris, Droz.

_ (1993): "Trois applications de la notion de verbe support", L'Information grammaticale, 59, pp. 16-22.

Gross, M. (1981): "Les bases empiriques de la notion de prédicat sémantique", Langages, 63, pp. 7-52.

(1994): "Constructing Lexicon-Grammars", en B. T. S. Atkins y A. Zampolli (eds.), Computational Approaches to the Lexicon, Oxford, Oxford University Press, pp. 213-263.

- (1998): "La fonction sémantique des verbes supports", Travaux de Linguistique, 37, pp. 25-46.

HaUsmann, F. (1979), "Un dictionnaire des collocations est-il possible?", Travaux de littérature et de linguistique de l'Université de Strasbourg, 17: 1, pp.187-195.

(1998): "Tout est idiomatique dans les langues", en M. MartinsBaltar (ed.), La locution entre langue et usages, Fontenay/St.Cloud, ENS., pp. 277-290.

HEID, U. (1992): "Décrire les collocations. Deux approches lexicographiques et leur application dans un outil informatisé", Terminologie et Traduction, 2: 3, pp. 523-548.

_ (1994): "On Ways Words Work Together. Topics in Lexical Combinatorics", Euralex'94 Proceedings, Amsterdam: Vrije Universiteit, pp. 226-257.

— (1996): "Using Lexical Functions for the Extraction of Collocations 
from Dictionaries and Corpora”, en L. Wanner (ed.), 1996, pp. 115146.

— y S. RAAB (1989): "Collocations in Multilingual Generation", en Proceedings of the 4th Meeting of the Association of Computational Linguistics, European Chapter.

Heylen, D. (1993): "Characterizing Collocations", Collocations ET10/75, vol. 1, pp. 13-34.

IBRAHIM, A. H. (ed.) (1996): Les supports, Langages, vol. 121.

JACKENDOFF, R. (1983): Semantics and Cognition, Cambridge, MIT Press.

(1990): Semantic Structures, Cambridge, MIT Press.

Jespersen, O. (1954): A Modern English Grammar on Historical Principles, London, George Allen \& Unwin.

KoIKE, K. (1992): "Locución verbal y verbo compuesto", Hispánica, 36, pp. 89-104.

- (1996-1997): Alcance y características de las colocaciones sustantivo-verbales en español, Memoria de licenciatura, Madrid, UNED.

LÉPINETTE, B. (1989): "Vers un dictionnaire explicatif et combinatoire bilingue: bases théoriques et élaboration de quelques articles", Cahiers de lexicologie, 54: 1, pp. 105-162.

LuQue Durán, J., y F. MANJón PozAs (1997a), "Un ejemplo de diccionario explicativo-combinatorio del español”, en J. Luque Durán y F. Manjón Pozas (eds.), Estudios de lexicología y creatividad léxica, Granada, Método Ediciones, pp. 147-154.

(1997b): "Los diccionarios explicativo-combinatorios de la Escuela Semántica de Moscú-Montreal”, en J. Luque Durán y A. Pamies Bertrán (eds.), Panorama de lexicología y lexicografia, Granada, Método Ediciones, pp. 55-71

MeL'ČUK, I. (1973): “Towards a Linguistic 'Meaning-Text' Model”, en F.

Kiefer (ed.), Trends in Soviet Theoretical Linguistics, Dordrecht, Reidel, pp. 33-57.

(1982): "Lexical Functions in Lexicographic Description", en Proceedings of the Eighth Annual Meeting of the Berkeley Linguistics Society, Berkeley, UCB, pp. 427-444.

- (1988a): Dependency Syntax: Theory and Practice, Albany, The State University of New York Press.

- (1988b): "Semantic Description of Lexical Units in an Explanatory Combinatorial Dictionary: Basic Principles and Heuristic Criteria", International Journal of Lexicography, 1: 1, pp. 1-13. 
(1993): Cours de morphologie générale, vol. 1, Montréal/Paris, Presses de l'Université de Montréal/Éditions du C.N.R.S.

- (1994): Cours de morphologie générale, vol. 2, Montréal/Paris, Presses de l'Université de Montréal/Éditions du C.N.R.S.

- (1995): "Phrasemes in Language and Phraseology in Linguistics", en M. Everaert, E. J. van der Linden, A. Schenk y R. Schrender (eds.), Idioms: Structural and Psychological Perspectives, Hillsdale/Hove, Lawrence Erlbaum Associates, pp. 167-232.

(1996a): Cours de morphologie générale, vol. 3, Montréal/Paris, Presses de l'Université de Montréal/Éditions du C.N.R.S.

- (1996b): "Lexical Functions: A Tool for the Description of Lexical Relations in the Lexicon", en L. Wanner (ed.), 1996, pp. 37-102.

— (1997a): Cours de morphologie générale, vol. 4, Montréal/Paris, Presses de l'Université de Montréal/Éditions du C.N.R.S.

_- (1997b): Vers une linguistique Sens-Texte, Paris, Collège de France. (1999): Communicative Organization of Sentences in Natural Language, Amsterdam, John Benjamins.

MEL'ČUK, I., N. ARBATCHEWSKY-JUMARIE, L. ELNITSKY, L. IORDANSKAJA y A. LESSARD (1984): Dictionnaire explicatif et combinatoire du français contemporain. Recherches lexico-sémantiques I, Montréal, Les Presses de l'Université de Montreál.

Mel'čuk, I., N. Arbatchewsky-Jumarie, L. Dagenais, L. Elnitsky, L. IORDANSKAJA, M. N. LEFEBVRE y S. MANTHA (1988): Dictionnaire explicatif et combinatoire du français contemporain. Recherches lexicosémantiques II, Montréal, Les Presses de l'Université de Montréal.

Mel'ČuK, I., N. Arbatchewsky-Jumarie, L. Iordanskaja y S. Manthạ (1992): Dictionnaire explicatif et combinatoire du français contemporain. Recherches lexico-sémantiques III, Montréal, Les Presses de l'Université de Montréal.

Mel'čuk, I., N. Arbatchewsky-Jumarie, L. Iordanskaja, S. Mantha y A. PolguÈRe (1999): Dictionnaire explicatif et combinatoire du français contemporain. Recherches lexico-sémantiques IV, Montréal, Les Presses de l'Université de Montréal.

Mel'čuK, I., A. Clas y A. Polguère (1995): Introduction à la lexicologie explicative et combinatoire, Louvain-la-Neuve, Duculot.

MEL'ČUK, I. y L. WANNER (1996): “Lexical Functions and Lexical Inheritance for Emotion Lexemes in German", en L. Wanner (ed.), 1996, pp. 209-279. 
MeL'ČUK, I. y A. Žolóvovskis (1970): “Towards a Functioning MeaningText Model of Language", Linguistics, 57, pp. 10-47.

RAPPAPORT, M. y B. LEVIN (1988): "What to do with $\theta$-Roles", en W. Wilkins (ed.), Thematic Relations, Syntax and Semantics 21, New York: Academic Press, pp. 7-36.

SANROMÁn, B. (1998): Contribución lexicográfica al estudio de los nombres de emoción, Memoria de licenciatura, Coruña, Universidade da Coruña.

_ (1998-1999); "La polisemia del nombre EsPERANZA: su delimitación en lexemas", Revista de Lexicografia, 5, pp. 157-174.

— - I. Lareo y M. Alonso Ramos (1999): "Transferencia léxica y reglas de paráfrasis: verbos denominales de SP cognado", Procesamiento del lenguaje natural, 25, pp.183-189.

SMADJA, F. A. (1993): "Retrieving collocations from text: Xtract", Computational Linguistics, 19:1, pp. 143-177.

SolÉ, Y. (1966): Hacer: verbo funcional y lexical, Ann Arbor, Georgetown University.

Somers, H. L. (1984): "On the Validity of the Complement-Adjunct Distinction in Valency Grammar", Linguistics, 22, pp. 507-530.

SteELE, J. (ed.) (1990): Meaning-Text Theory: Linguistics, Lexicography and Implications, Ottawa, University of Ottawa Press.

— e I. Meyer (1990): "Lexical Functions in an Explanatory Combinatorial Dictionary: Kinds, Descriptions, and English Examples", en J. Steele (ed.), pp. 41-61.

WANNER, L. (ed.) (1996): Lexical Functions in Lexicography and Natural Language Processing, Amsterdam/Philadelphia: John Benjamins.

- (ed.) (1997): Recent Trends in Meaning-Text Theory, Amsterdam/Philadelphia, John Benjamins.

WotJAK, G. (1998): "Reflexiones acerca de construcciones verbo-nominales funcionales", en G. Wotjak (ed.), Estudios de fraseología y fraseografia del español actual, Frankfurt/Madrid, Vervuert/Iberoamericana, pp. 257-279. 